\title{
Correction to: Outcomes associated with immune-related adverse events in metastatic non-small cell lung cancer treated with nivolumab: a pooled exploratory analysis from a global cohort
}

\author{
Abdul Rafeh Naqash ${ }^{1,7}$. Biagio Ricciuti ${ }^{2,3}$. Dwight H. Owen ${ }^{4}$. Vaia Florou ${ }^{5,10} \cdot$ Yukihiro Toi $^{6} \cdot$ Cynthia Cherry $^{1,7}$. \\ Maida Hafiz ${ }^{8}$. Andrea De Giglio ${ }^{2}$. Mavish Muzaffar ${ }^{7}$. Sandip H. Patel ${ }^{4}$. Shunichi Sugawara ${ }^{6}$. Jarred Burkart ${ }^{4}$. \\ Wungki Park ${ }^{5,9} \cdot$ Rita Chiari $^{2} \cdot$ Jun Sugisaka ${ }^{6}$. Gregory A. Otterson ${ }^{4} \cdot$ Gilberto de Lima Lopes $^{5} \cdot$ Paul R. Walker $^{7}$
}

Published online: 28 April 2020

(c) This is a U.S. government work and not under copyright protection in the U.S.; foreign copyright protection may apply 2020

\section{Correction to: Cancer Immunology, Immunotherapy https://doi.org/10.1007/s00262-020-02536-5}

The original version of this article unfortunately contained a mistake. The second sentence of the section "irAEs and ICI efficacy" should read as

Patients with irAEs had a higher objective response rate (ORR) compared with patients with no-irAEs $40.1 \%$ (4.9\% $\mathrm{CR}+35.2 \% \mathrm{PR})$ versus $14.1 \%[(2.1 \% \mathrm{CR}+12.1 \%$ $\mathrm{PR}) ; p<0.01]$.
Publisher's Note Springer Nature remains neutral with regard to jurisdictional claims in published maps and institutional affiliations.

The original article can be found online at https://doi.org/10.1007/ s00262-020-02536-5.

Abdul Rafeh Naqash

abdulrafeh.naqash@nih.gov; rafehnaqash@ hotmail.com

1 Division of Cancer Treatment and Diagnosis, Developmental Therapeutics Clinic, National Cancer Institute, Bldg 31/3A44, 31 Centre Drive, Bethesda, MD 20892, USA

2 Thoracic Oncology Unit, Santa Maria Della Misericordia Hospital, University of Perugia, Perugia, Italy

3 Lowe Center for Thoracic Oncology, Dana-Farber Cancer Institute, Boston, MA, USA

4 Division of Medical Oncology, Department of Internal Medicine, Ohio State University, Columbus, OH, USA
5 Division of Oncology, Department of Medicine, University of Miami Miller School of Medicine, Miami, FL, USA

6 Department of Pulmonary Medicine, Sendai Kousei Hospital, Hirosemachi, Aoba-Ku, Sendai, Japan

7 Division of Hematology/Oncology, Department of Internal Medicine, East Carolina University, Greenville, NC, USA

8 Department of Pulmonary Medicine, East Carolina University, Greenville, NC, USA

9 Memorial Sloan Kettering Cancer Center, New York, USA

10 Division of Medical Oncology, Huntsman Cancer Institute, University of Utah, Salt Lake City, UT, USA 